\title{
Neutrophil Cd64 as a Diagnostic Marker in Neonatal Sepsis
}

\author{
Iris Streimish, MD*, ${ }^{*}$ Matthew Bizzarro, MD*, Veronika Northrup, MPH ${ }^{\#}$, Chao Wang, BS ${ }^{\star *}$, \\ Sara Renna, PA ${ }^{\star}$, Nancy Koval, NNP*, Fang-Yong Li, MPH", Richard Ehrenkranz, MD*, \\ Henry M. Rinder, MD ${ }^{\star *}$, and Vineet Bhandari, MD, $\mathbf{D M}^{\star}, \S$ \\ "Division of Perinatal Medicine, Department of Pediatrics, Yale University School of Medicine, \\ New Haven, CT \\ ${ }^{*}$ Division of Perinatal Medicine, Department of Laboratory Medicine, Yale University School of \\ Medicine, New Haven, CT \\ \#Yale Center for Analytical Sciences, New Haven, CT, USA
}

\begin{abstract}
We assessed neutrophil CD64 as a diagnostic marker for neonatal sepsis. For early-onset sepsis, the CD64 index with a cut-point value of 2.38 had sensitivity, specificity and a negative predictive values of $100 \%, 68 \%$, and $100 \%$, respectively. For late-onset sepsis, the respective values were $3.62,75 \%, 77 \%$, and $96 \%$. Neutrophil CD64 index can be incorporated as a valuable marker for excluding neonatal sepsis.
\end{abstract}

\section{Keywords}

Sensitivity; specificity; infection; newborn; CD64; diagnosis of sepsis

\section{INTRODUCTION}

Sepsis continues to be a major source of morbidity and mortality in the neonatal population. ${ }^{1-3}$ Early-onset sepsis (EOS), diagnosed $\leq 72$ hours after birth, is most often related to antenatal and perinatal factors including prolonged rupture of amniotic membranes, maternal colonization with group B streptococcus (GBS), and maternal chorioamnionitis. ${ }^{2}$ Late-onset sepsis (LOS), diagnosed $>72$ hours after birth, is primarily hospital acquired and occurs more commonly in preterm infants. ${ }^{3,4}$ Major risk factors for LOS include prolonged mechanical ventilation, ${ }^{3}$ the use of indwelling vascular catheters, ${ }^{3}$ and necrotizing enterocolitis. ${ }^{3,5}$

Early recognition and timely institution of antimicrobials in infants at risk for sepsis is of utmost importance as delayed or missed treatment can have serious consequences. ${ }^{6}$ As a result, evaluations for sepsis in combination with empiric broad-spectrum antimicrobial treatment while awaiting culture results (which typically take about 2 days) are a common

\footnotetext{
$\S$ Address for correspondence: Vineet Bhandari, MD, DM, Yale University School of Medicine, Yale Child Health Research Center, Room Number: 219, P.O. Box 208081, 464 Congress Avenue, New Haven, CT 06520, Phone: 203-785-2613, Fax: 203-737-5872, vineet.bhandari@yale.edu.

Current affiliation: Section of Neonatology, Department of Pediatrics, Newton Wellesley Hospital, Special Care Nursery, Newton, MA 02462

Publisher's Disclaimer: This is a PDF file of an unedited manuscript that has been accepted for publication. As a service to our customers we are providing this early version of the manuscript. The manuscript will undergo copyediting, typesetting, and review of the resulting proof before it is published in its final citable form. Please note that during the production process errors may be discovered which could affect the content, and all legal disclaimers that apply to the journal pertain.
} 
practice in the newborn intensive care unit (NICU). 1,2,6 This practice may also result in admission to a NICU and separation from parents, as well as increased costs to the healthcare system. In addition, concerns have been raised that increased antibiotic exposure contributes to resistance among microorganisms as well as alterations in the normal microbiota of exposed neonates. ${ }^{6-8}$

The current gold standard for the diagnosis of neonatal sepsis is blood culture. In addition to the time required to confirm blood culture results, the test itself can often be unreliable. ${ }^{2,9}$ This test, combined with a lack of specific clinical signs for infections in neonates, often makes diagnosing and determining an appropriate duration of antimicrobial therapy for neonatal sepsis difficult. ${ }^{2,6,9}$ A more expedient, sensitive, and specific diagnostic tool for bloodstream infections is therefore needed. Several hematologic markers for infection, such as C-reactive protein (CRP) and procalcitonin, have been investigated alone and in combination with other clinical and laboratory data, with mixed results. ${ }^{9-15}$ One marker that has shown particular promise as an early marker for infection is neutrophil surface CD64 expression. ${ }^{13,16,17} \mathrm{CD} 64$ is a high affinity $\mathrm{Fc}$ receptor expressed on neutrophils. Expression increases when neutrophils are activated by infectious stimuli. We conducted a prospective investigation aimed at assessing the sensitivity and specificity of neutrophil CD64 as a marker for bloodstream infection in infants undergoing a sepsis evaluation in the NICU at Yale-New Haven Children's Hospital.

\section{MATERIALS AND METHODS}

This study was approved by the Yale University School of Medicine Human Investigation Committee and conducted in the NICU at Yale-New Haven Children's Hospital.

\section{Patient Population}

We enrolled consecutive patients from January 2008 through June 2009 who underwent a sepsis evaluation in the NBSCU. This evaluation typically included a complete blood count (CBC), two peripheral blood cultures and, particularly for LOS, urine and cerebrospinal fluid cultures. Each evaluation represented a separate episode of suspected sepsis for each neonate. A patient could be enrolled multiple times, for each separate sepsis evaluation performed during their NBSCU admission. Patients were excluded (1) if they did not have a CBC performed at or up to 24 hours prior to obtaining blood cultures and/or (2) if a CD64 index was not performed within 48 hours of obtaining the CBC. Neutrophil CD64 values have been reported to be sustained for at least 24 hours in neonates with sepsis. ${ }^{18}$

\section{Data Collection}

Data were obtained by accessing the electronic medical record. Laboratory data on each patient admitted to the NBSCU was checked every 1 to 3 days. If a patient had a blood culture(s) and a CBC at or up to 24 hours prior to obtaining blood cultures, a CD64 index was performed on the same sample of blood used for the CBC. Additional patient-related data were obtained from the same electronic system. Antenatal and perinatal data collection included: (1) birth weight (BW), which was classified into three categories: very low BW (VLBW; $\leq 1500 \mathrm{~g}$ ), LBW (1500 g - $2500 \mathrm{~g}$ ), and normal (above $2500 \mathrm{~g}$ ); (2) gestational age, (GA); (3) small for GA status (i.e. BW <10 th percentile for GA); ${ }^{19}$ (4) maternal GBS colonization status; (5) clinical or culture-proven chorioamnionitis (based on delivering obstetrician's diagnosis); (6) maternal intrapartum antibiotic exposure (including type, rationale for use, and duration of exposure prior to delivery categorized as < or $\geq 4$ hours); (7) antenatal glucocorticoid administration and timing of last dose prior to delivery; and (8) placental histology. 
Data related to the infant's hospital course included (1) presence of patent ductus arteriosus (diagnosed via echocardiographic evaluation by a pediatric cardiologist), (2) diagnosis of necrotizing enterocolitis ( $\geq$ Bell's Stage II) ${ }^{20}$ (3) diagnosis of bronchopulmonary dysplasia (defined as oxygen treatment at 36 weeks post menstrual age); (4) duration of respiratory support (including mechanical ventilation and need for supplemental oxygen); (5) death; and (6) length of hospitalization.

Information about each sepsis evaluation included: (1) day of life that the evaluation was performed, (2) laboratory data results, (3) final diagnosis for type of sepsis, and (4) specific antimicrobial therapy and its duration of use.

\section{Sepsis Evaluations}

In general, the clinical and historical features used to identify patients at risk for sepsis included one or more of the following, as determined by the attending neonatologist: ${ }^{21-23}$ (1) respiratory compromise (e.g. tachypnea, increase in frequency or severity of apnea, increased ventilator support); (2) cardiovascular compromise (e.g. increase frequency or severity of bradycardic episodes, pallor, decreased perfusion, hypotension); (3) metabolic changes (e.g. temperature instability, feeding intolerance, glucose instability, metabolic acidosis); (4) neurologic changes (e.g. lethargy, hypotonia, irritability); and (5) antenatal risk factors (e.g. maternal GBS colonization without adequate intrapartum prophylaxis, unknown maternal GBS status, maternal temperature, chorioamnionitis, preterm labor, and prolonged rupture of membranes).

All blood cultures were collected using standard sterile techniques. As per unit protocol, we attempt to obtain 2 blood cultures with a minimum of $0.5 \mathrm{ml}$. The BACTEC ${ }^{\circledR}$ (Becton Dickinson and Co., Sparks, MD) microbial detection system was used to detect positive blood cultures.

\section{CD64 Expression}

As previously published, neutrophil CD64 expression was measured using $50 \mu \mathrm{L}$ of whole blood incubated for 10 minutes at room temperature with a saturating amount of fluorescein isothiocyanate (FITC)-conjugated anti-CD64 monoclonal antibody or isotype control (Leuko64 kit, Trillium Diagnostics, Scarborough, ME), followed by ammonium chloridebased red cell lysis. Samples were washed once and re-suspended in 0.5 milliliters of phosphate-buffered saline with $0.1 \%$ bovine serum albumin. Flow cytometric analysis was accomplished using a Becton-Dickinson FACScan (Mountainview, CA) to collect log FITC fluorescence, log right-angle side scatter and forward scatter on a minimum of 50,000 leukocytes. Interassay standardization and CD64 quantitation were performed using FITC calibration beads (Leuko64 kit). Data analysis was performed using light scatter gating to define the neutrophil population, and neutrophil CD64 Index was quantified as mean equivalent soluble fluorescence units using QuickCal for Winlist (Verity Software House, Topsham, ME) with a correction for nonspecific antibody binding by subtracting values for the isotype control. ${ }^{13}$ This was expressed as an absolute value. The intra-assay coefficient of variation of the neutrophil CD64 Index was $<3 \%$ while the inter-assay coefficient of variation was $<5 \%$. Investigators (CW, HMR) checking and confirming the neutrophil CD64 results were blinded to the clinical data, including the blood culture results. Clinicians did not have access to the neutrophil CD64 values and these were not used to decide initiation or duration of antibiotic therapy. 


\section{Defining Sepsis as Outcome}

Individual sepsis evaluations with positive blood cultures were diagnosed as culture-proven sepsis according to the current National Healthcare Safety Network definitions for laboratory-confirmed bloodstream infections. ${ }^{24}$

\section{Statistical analyses}

Data were analyzed for the definition of sepsis (culture-proven), and then further stratified by EOS and LOS. To account for the correlation between repeated evaluations within same infant, the generalized linear mixed modeling (GLMM) approach was used to predict the probability of sepsis. First, individual risk factors were evaluated in bivariate analyses, and the significant associations were then entered into a multivariate model which consisted of CD64 index as the main predictor variable and categorical birth weight as an adjustment variable. The goodness-of-fit was tested using the likelihood ratio test. Different covariance structures were compared in order to choose the best fit. Compound symmetry (CS) was chosen for the final models based on the Akaike Information Criterion (AIC), and because unstructured covariance caused convergence problems, while independent structure ignored the within-subject correlations.

Receiver operating characteristic (ROC) curves were constructed using the predicted probability of sepsis from GLMM. The ROC curve is a plot of 1-specificity (1-Sp) versus sensitivity (Se) at all observed values of CD64. In order to quantify the predictive value of CD64 index, we calculated the area under the ROC curve (AUC), and then identified the optimum cut-point of CD64 index based on maximizing the Youden Index ( $J=$ $\left.\max \left[S E_{(C D 64)}+S P_{(C D 64)}-1\right]\right)$. The cut-point corresponds to the optimal differentiating ability of CD64 when equal weights are given to sensitivity and specificity. As BW was a significant predictor of probability of overall culture-proven sepsis (EOS and LOS combined), we calculated CD64 cut-points for the three categories of birth weight.

These analyses were conducted using a modified SAS macro. ${ }^{25}$ Statistical significance was established at alpha $=0.05$. Another macro was created to estimate the standard error of AUC using a bootstrapping approach for 250 samples, which was then used to obtain $95 \%$ confidence interval for AUC. These analyses were performed with SAS 9.2 (Cary, NC). Sensitivity, specificity, positive predictive value (PPV), negative predictive value (NPV) and likelihood ratios at the optimum cut-off CD64 index points were calculated using appropriate formulas in Microsoft Excel (Redmond, WA).

\section{RESULTS}

1383 sepsis evaluations were performed in 749 infants during the study period. The majority of infants (71.4\%) had only one evaluation, $19.2 \%$ had 2 or 3 evaluations, and $9.4 \%$ had $>3$ sepsis evaluations performed during their hospitalization. One case of EOS, which was positive for Candida albicans had the highest CD64 value (51.94) noted in our study. Since the value was so extreme, it significantly skewed the CD64 distribution. Hence, the patient was excluded from subsequent analyses noted in the Tables, Supplemental Digital Content 1 and 2, http://links.lww.com/INF/B179, http://links.lww.com/INF/B180 and Figure 1.

\section{Culture-Proven Sepsis}

Blood culture and CD64 data were available on 997 sepsis evaluations (excluding the EOS case mentioned above) done in 649 infants, of which $50(5 \%)$ instances of positive culture in the culture-proven sepsis category were identified. Three of these cases were early-onset in nature. The responsible organisms were determined to be GBS ( 2 cases) and E. coli ( 1 case). Forty-seven cases were categorized as late-onset. There were 37 cases of monomicrobial 
LOS and 10 cases of polymicrobial LOS. Responsible organisms for monomicrobial sepsis included species of Coagulase-negative Staphylococci (CONS; 9 cases), Staphylococcus aureus ( 9 cases), E. coli (6 cases), Enterococcus (5 cases), Candida albicans ( 2 cases), Candida parapsilosis (2 cases), Pseudomonas aeruginosa (2 cases), GBS (1 case) and Leclercia adecarboxylata ( 1 case). Polymicrobial cases consisted of 6 cases of Klebsiella pneumoniae and Enterococcus fecalis and 4 cases of Entercoccus and CONS.

Demographic and clinical characteristics of infants by positive and negative sepsis evaluations are presented in Table, Supplemental Digital Content 1, http://links.lww.com/INF/B179. Out of a total of 40 deaths, there were 6 deaths among those who had at least one positive blood culture. Data on histological chorioamnionitis were available on 339/649 (52.2\%) infants in the "culture-proven sepsis" category and were not significantly different between the "positive" and "negative" sepsis groups. BW category, gestational age, antenatal steroids, ventilation days, length of stay and CD64 were identified as significant factors associated with culture proven sepsis in the unadjusted analyses ( $\mathrm{p}$ $<0.01$ for all).

In the adjusted analyses, only BW and CD64 index were determined to be significant predictors of overall (i.e. both EOS and LOS) culture proven sepsis ( $p<0.01$ and $p<0.0001$ respectively). The ROC curve analyses showed that the CD64 index had an AUC of 0.87 for the overall proven sepsis (Figure 1). The optimum CD64 cut-point was 3.57, 3.75, and 4.61 for VLBW, LBW and normal BW, respectively.

Within EOS, CD64 index did not reach statistical significance ( $\mathrm{p}=0.11$ ), which was likely due to the small sample size; however it remained highly predictive of culture-proven LOS $(\mathrm{p}<0.0001)$. For EOS, sensitivity and specificity of the CD64 index with a cut-point value of 2.38 were determined to be $100 \%$ and $68 \%$, respectively, with a NPV of $100 \%$. For LOS, a cut-point value of 3.62 yielded a sensitivity, specificity, and NPV value of $75 \%, 77 \%$, and 96\%, respectively (Table, Supplemental Digital Content 2, http://links.lww.com/INF/B180).

\section{DISCUSSION}

The potential for significant adverse consequences associated with neonatal sepsis often result in a low threshold for evaluation and institution of antimicrobial therapy. In such circumstances the usual clinical practice in an infant with suspected sepsis is to obtain hematologic studies and blood cultures prior to the initiation of empiric antibiotics. ${ }^{6}$ Conservative estimates suggest that at least $70 \%$ of infants admitted to NICUs undergo at least one sepsis evaluation. ${ }^{7,26}$ In the majority of cases, the results of the tests are negative and antimicrobial therapy is discontinued in $48-72 \mathrm{~h} .{ }^{1,6,7}$ Sepsis evaluations thus may result in a period of admission to a NICU for at least $48 \mathrm{~h}$ of observation while awaiting blood culture results. ${ }^{1,6}$ Since the highest estimates of blood culture positive rates in the NICU only approach $20 \%,{ }^{27}$ this suggests that a very high number of infants are unnecessarily exposed to antimicrobials.

If clinicians follow the $48 \mathrm{~h}$ "rule out sepsis" approach, strictly based on blood culture results, this will cause unnecessary separation of infant and family, an increased length of hospital stay and enhanced costs. ${ }^{28}$ In addition, if the clinicians decide to continue empiric antibiotics in the presence of a negative blood culture, such antimicrobial overexposure may promote antimicrobial resistance among certain strains of bacteria and fungi. ${ }^{7}$ Furthermore, prolonged exposure to antibiotics in the neonatal period can alter the infant's normal microbial flora which may subsequently predispose them to necrotizing enterocolitis. 8,29 The extent of this problem can be surmised from the fact that infants with "clinical sepsis" 
may include $20 \%-90 \%$ of patients who undergo sepsis evaluations and treatment in NICUs. ${ }^{29}$

Thus, the need for a supplemental test for the diagnosis of neonatal sepsis with high sensitivity and specificity is readily apparent. ${ }^{9,28,30}$ Such a test needs to be reliable, rapid, and ideally require a minimal amount of blood. Currently, most clinicians rely on the CBC and its derived indices, including the absolute neutrophil count, the absolute band count and the immature:total neutrophil ratio. ${ }^{9,} 31$ Individually they do not possess high specificity or sensitivity, and are generally more helpful when considered together; ${ }^{9}, 28,30,31$ however, these indices may be unreliable in the first few hours after birth or in the early stages of infection.

Among other proposed markers, procalcitonin, an acute phase reactant, has shown the most promise in detecting sepsis in the neonatal population. ${ }^{12,30,32}$ The primary disadvantages of procalcitonin include the need for GA-specific norms, the 4-6h required before elevation can be detected, and its relative lack of specificity. ${ }^{9,28,32}$ In addition, this testing platform is not readily available in all institutions. C-reactive protein is a well-studied acute phase reactant, more helpful for guiding duration of antibiotic therapy, rather than making the diagnosis of sepsis. This particular test is best used in LOS, requires serial measurements to provide the best sensitivity and specificity, and is utilized for its high NPV. $9,11,28,30$ Various cytokines have also been evaluated, although their potential widespread use is limited by their inconsistent cutoff values, delay in getting results, and lack of availability for routine measurements in clinical laboratories. ${ }^{21,28,30}$

We sought to evaluate the neutrophil CD64 index as a possible hematologic marker of sepsis for a variety of reasons. First, several studies have investigated the usefulness of this index in the NICU population, albeit in much smaller cohorts, but with promising results in both the preterm and term populations, as well as in cases of both EOS and LOS. ${ }^{13,30,33-37}$ Secondly, testing can be done on the same sample sent for a $\mathrm{CBC}$ evaluation as it requires only $50 \mu \mathrm{l}$ of blood. Thirdly, the CD64 results can be made available within hours of the $\mathrm{CBC}$, since most clinical laboratories in the developed and some developing countries have flow cytometry technology. Furthermore, standard cell counters which use flow cytometry have the potential to incorporate anti-CD64 antibodies and software to provide an even more rapid enumeration of CD64 indices nearly simultaneous with $\mathrm{CBC}$ results.

In our present study, we prospectively evaluated almost 1400 sepsis evaluations, and found that for culture-proven sepsis, using a cut-point CD64 Index value of 2.38 for EOS, the test had a sensitivity of $100 \%$, a specificity of $68 \%$, and a NPV of $100 \%$; while using a cut-point value of 3.62 for LOS sepsis, the test had a sensitivity of $75 \%$, a specificity of $77 \%$, and a NPV of $96 \%$. The AUC was 0.87 overall, with higher AUC for both EOS and LOS. Given the high NPV value of CD64, it has strong potential to influence the initiation, early termination and duration of antibiotic therapy. If CD64 values remain below the cut-offs noted, our data suggests the potential to use this information to discontinue antibiotics.

This study does have some limitations. We did not have complete information on the histological presence of chorioamnionitis, since only selective placentae were sent for histopathological examination. The number of positive cultures in EOS was small. In addition, when the analyses were stratified by the BW categories, some of the strata had small number of cases, and that would limit the generalizability of the findings for BW. Despite these shortcomings, we were able to show that some common clinical variables (e.g. mode of delivery, exposure to antenatal steroids) did not impact on the association between culture-proven sepsis and CD64 Index. 
The strengths of our study include its prospective nature, the largest sample size to date in the neonatal population, the detailed collection of clinical data, and the reasonable number of proven sepsis cases.

We believe that neutrophil CD64 Index should be incorporated as a useful marker for excluding the diagnosis of neonatal sepsis, given its high negative predictive value for culture-proven infection. Further research should focus on the cost-effectiveness of CD64 and its utility for antibiotic stewardship - i.e. for initiating as well as terminating antibiotic therapy - in the vulnerable NICU population.

\section{Supplementary Material}

Refer to Web version on PubMed Central for supplementary material.

\section{Acknowledgments}

Biostatistical collaboration was provided through CTSA Grant Number UL1 RR024139 from the National Center for Research Resources (NCRR), a component of the National Institutes of Health (NIH), and NIH Roadmap for Medical Research. Its contents are solely the responsibility of the authors and do not necessarily represent the official view of NCRR or NIH. Information on Re-engineering the Clinical Research Enterprise can be obtained from the NIH website. This work was supported in part by an unrestricted educational grant from Trillium Diagnostics to HMR. Trillium Diagnostics and its employees were not involved in the design and conduct of the study; collection, management, analysis, and interpretation of the data; and preparation, review, or approval of the manuscript.

No honorarium, grant, or other form of payment was given to anyone to produce the manuscript.

We thank the NICU nurses at the Yale-New Haven Children's Hospital for their invaluable assistance.

\section{REFERENCES}

1. Garcia-Prats JA, Cooper TR, Schneider VF, Stager CE, Hansen TN. Rapid detection of microorganisms in blood cultures of newborn infants utilizing an automated blood culture system. Pediatrics. 2000; 105:523-527. [PubMed: 10699103]

2. Ganatra HA, Stoll BJ, Zaidi AK. International perspective on early-onset neonatal sepsis. Clin Perinatol. 2010; 37:501-523. [PubMed: 20569819]

3. Stoll BJ, Hansen N, Fanaroff AA, et al. Late-onset sepsis in very low birth weight neonates: the experience of the NICHD Neonatal Research Network. Pediatrics. 2002; 110:285-291. [PubMed: 12165580]

4. Bizzarro MJ, Raskind C, Baltimore RS, Gallagher PG. Seventy-five years of neonatal sepsis at Yale: 1928-2003. Pediatrics. 2005; 116:595-602. [PubMed: 16140698]

5. Patel S, Dammann O, Martin CR, Allred EN, Leviton A. Presumed and definite bacteremia in extremely low gestational age newborns. Acta Paediatr. 2011; 100:36-41. [PubMed: 20712830]

6. Kumar Y, Qunibi M, Neal TJ, Yoxall CW. Time to positivity of neonatal blood cultures. Arch Dis Child Fetal Neonatal Ed. 2001; 85:F182-F186. [PubMed: 11668160]

7. Bizzarro MJ, Gallagher PG. Antibiotic-resistant organisms in the neonatal intensive care unit. Semin Perinatol. 2007; 31:26-32. [PubMed: 17317424]

8. Alexander VN, Northrup V, Bizzarro MJ. Antibiotic exposure in the newborn intensive care unit and the risk of necrotizing enterocolitis. J Pediatr. 2011; 159:392-397. [PubMed: 21489560]

9. Benitz WE. Adjunct laboratory tests in the diagnosis of early-onset neonatal sepsis. Clin Perinatol. 2010; 37:421-438. [PubMed: 20569816]

10. Pierrakos C, Vincent JL. Sepsis biomarkers: a review. Crit Care. 2010; 14:R15. [PubMed: 20144219]

11. Benitz WE, Han MY, Madan A, Ramachandra P. Serial serum C-reactive protein levels in the diagnosis of neonatal infection. Pediatrics. 1998; 102:E41. [PubMed: 9755278] 
12. Fendler WM, Piotrowski AJ. Procalcitonin in the early diagnosis of nosocomial sepsis in preterm neonates. J Paediatr Child Health. 2008; 44:114-118. [PubMed: 17927729]

13. Bhandari V, Wang C, Rinder C, Rinder H. Hematologic profile of sepsis in neonates: neutrophil CD64 as a diagnostic marker. Pediatrics. 2008; 121:129-134. [PubMed: 18166566]

14. Okascharoen C, Sirinavin S, Thakkinstian A, Kitayaporn D, Supapanachart S. A bedside prediction-scoring model for late-onset neonatal sepsis. J Perinatol. 2005; 25:778-783. [PubMed: 16281050]

15. Rodwell RL, Taylor KM, Tudehope DI, Gray PH. Hematologic scoring system in early diagnosis of sepsis in neutropenic newborns. Pediatr Infect Dis J. 1993; 12:372-376. [PubMed: 8327296]

16. Ng PC, Li G, Chui KM, et al. Neutrophil CD64 is a sensitive diagnostic marker for early-onset neonatal infection. Pediatr Res. 2004; 56:796-803. [PubMed: 15371562]

17. Ng PC, Li K, Wong RP, Chui KM, Wong E, Fok TF. Neutrophil CD64 expression: a sensitive diagnostic marker for late-onset nosocomial infection in very low birthweight infants. Pediatr Res. 2002; 51:296-303. [PubMed: 11861933]

18. Layseca-Espinosa E, Perez-Gonzalez LF, Torres-Montes A, et al. Expression of CD64 as a potential marker of neonatal sepsis. Pediatr Allergy Immunol. 2002; 13:319-327. [PubMed: 12431190]

19. Alexander GR, Himes JH, Kaufman RB, Mor J, Kogan M. A United States national reference for fetal growth. Obstet Gynecol. 1996; 87:163-168. [PubMed: 8559516]

20. Bell MJ, Ternberg JL, Feigin RD, et al. Neonatal necrotizing enterocolitis. Therapeutic decisions based upon clinical staging. Ann Surg. 1978; 187:1-7. [PubMed: 413500]

21. Gonzalez BE, Mercado CK, Johnson L, Brodsky NL, Bhandari V. Early markers of late-onset sepsis in premature neonates: clinical, hematological and cytokine profile. J Perinat Med. 2003; 31:60-68. [PubMed: 12661146]

22. Klinger G, Levy I, Sirota L, Boyko V, Reichman B, Lerner-Geva L. Epidemiology and risk factors for early onset sepsis among very-low-birth-weight infants. Am J Obstet Gynecol. 2009; 201(38):e31-e36.

23. Benitz WE, Gould JB, Druzin ML. Risk factors for early-onset group B streptococcal sepsis: estimation of odds ratios by critical literature review. Pediatrics. 1999; 103:e77. [PubMed: 10353974]

24. Horan TC, Andrus M, Dudeck MA. CDC/NHSN surveillance definition of health care-associated infection and criteria for specific types of infections in the acute care setting. Am J Infect Control. 2008; 36:309-332. [PubMed: 18538699]

25. Liu H, Wu HC. Estimating the area under a receiver operating characteristic (ROC) curve for repeated measures design. J Stat Soft. 2003; 12:1-18.

26. Clark RH, Bloom BT, Spitzer AR, Gerstmann DR. Empiric use of ampicillin and cefotaxime, compared with ampicillin and gentamicin, for neonates at risk for sepsis is associated with an increased risk of neonatal death. Pediatrics. 2006; 117:67-74. [PubMed: 16396862]

27. Stoll BJ, Hansen N. Infections in VLBW infants: studies from the NICHD Neonatal Research Network. Semin Perinatol. 2003; 27:293-301. [PubMed: 14510320]

28. Mishra UK, Jacobs SE, Doyle LW, Garland SM. Newer approaches to the diagnosis of early onset neonatal sepsis. Arch Dis Child Fetal Neonatal Ed. 2006; 91:F208-F212. [PubMed: 16632649]

29. Cotten CM, Taylor S, Stoll B, et al. Prolonged duration of initial empirical antibiotic treatment is associated with increased rates of necrotizing enterocolitis and death for extremely low birth weight infants. Pediatrics. 2009; 123:58-66. [PubMed: 19117861]

30. Ng PC, Lam HS. Biomarkers for late-onset neonatal sepsis: cytokines and beyond. Clin Perinatol. 2010; 37:599-610. [PubMed: 20813273]

31. Rodwell RL, Leslie AL, Tudehope DI. Early diagnosis of neonatal sepsis using a hematologic scoring system. J Pediatr. 1988; 112:761-767. [PubMed: 3361389]

32. Auriti C, Fiscarelli E, Ronchetti MP, et al. Procalcitonin in detecting neonatal nosocomial sepsis. Arch Dis Child Fetal Neonatal Ed. 2011 Mar 15. [E-pub ahead of print].

33. Morsy AA, Elshall LY, Zaher MM, Abd Elsalam M, Nassr AE. CD64 cell surface expression on neutrophils for diagnosis of neonatal sepsis. Egypt J Immunol. 2008; 15:53-61. [PubMed: 20306688] 
34. Groselj-Grenc M, Ihan A, Derganc M. Neutrophil and monocyte CD64 and CD163 expression in critically ill neonates and children with sepsis: comparison of fluorescence intensities and calculated indexes. Mediators Inflamm. 2008; 2008:202646. [PubMed: 18604302]

35. Groselj-Grenc M, Ihan A, Pavcnik-Arnol M, Kopitar AN, Gmeiner-Stopar T, Derganc M. Neutrophil and monocyte CD64 indexes, lipopolysaccharide-binding protein, procalcitonin and Creactive protein in sepsis of critically ill neonates and children. Intensive Care Med. 2009; 35:1950-1958. [PubMed: 19756501]

36. Zeitoun AA, Gad SS, Attia FM, Abu Maziad AS, Bell EF. Evaluation of neutrophilic CD64, interleukin 10 and procalcitonin as diagnostic markers of early-and late-onset neonatal sepsis. Scand J Infect Dis. 2010; 42:299-305. [PubMed: 20085423]

37. Dilli D, Oguz SS, Dilmen U, Koker MY, Kizilgun M. Predictive values of neutrophil CD64 expression compared with interleukin- 6 and C-reactive protein in early diagnosis of neonatal sepsis. J Clin Lab Anal. 2010; 24:363-370. [PubMed: 21089165] 


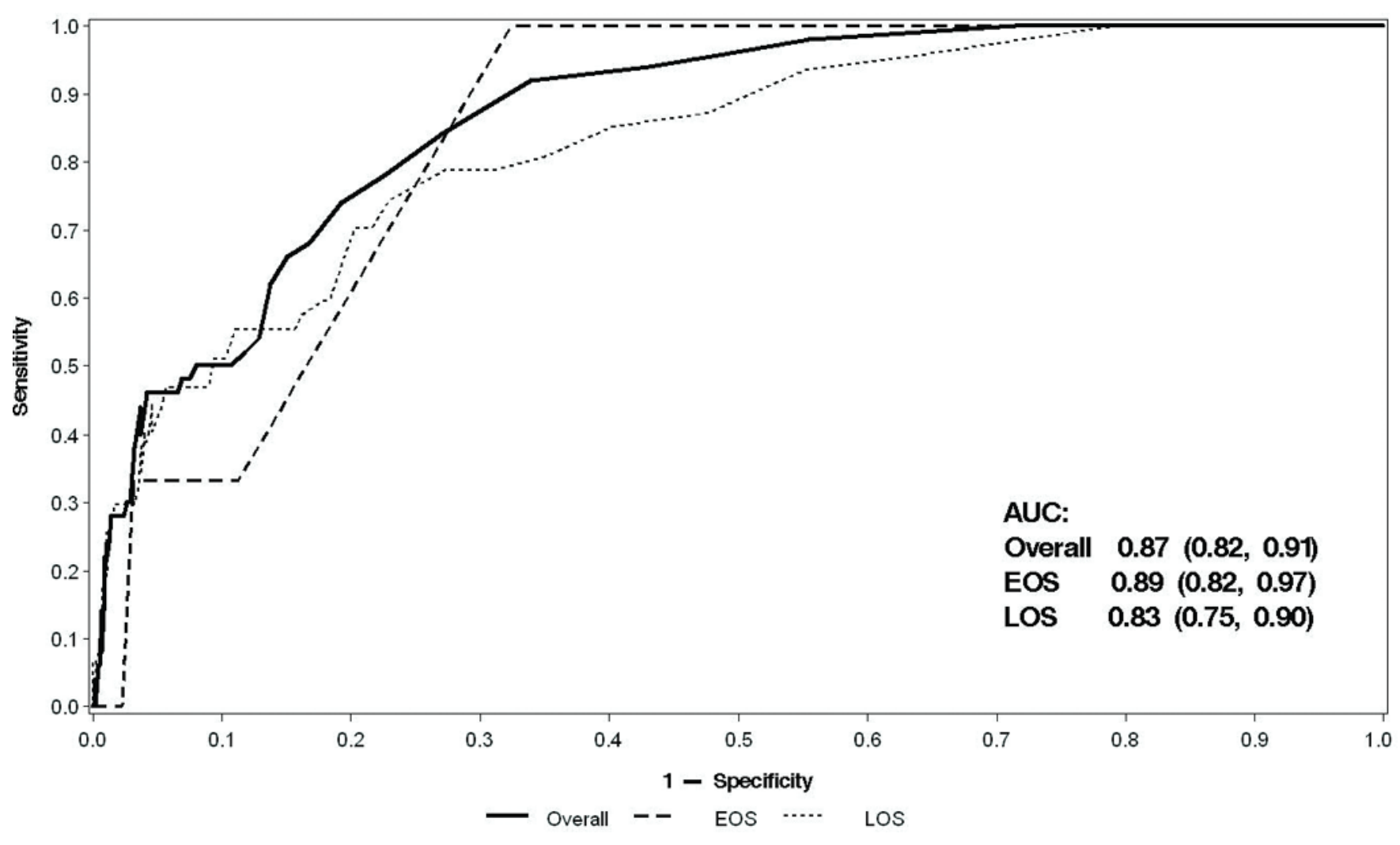

Figure 1.

Area under the receiver operating characteristic (ROC) curve (AUC) values $(95 \% \mathrm{CI})$ of CD64 Index for overall, EOS and LOS culture-proven sepsis.

EOS: early-onset sepsis; LOS: late-onset sepsis. 